\title{
Chirality induction on non-chiral dye-linked polysilsesquioxane in nanohelical structures
}

Received 00th January 20xx, Accepted 00th January 20xx DOI: $10.1039 / x 0 x \times 00000 x$

\author{
Naoya Ryu, ${ }^{\text {a }}$ Tsutomu Kawaguchi, ${ }^{\text {a }}$ Hiroshi Yanagita, ${ }^{\mathrm{b}}$ Yutaka Okazaki, ${ }^{\mathrm{c}}$ Thierry Buffeteau, ${ }^{\mathrm{d}}$ \\ Kyohei Yoshida, ${ }^{\mathrm{e}}$ Tomohiro Shirosaki, ${ }^{a}$ Shoji Nagaoka, ${ }^{\mathrm{ab}}$ Makoto Takafuji, ${ }^{\mathrm{b}}$ Hirotaka Ihara* ${ }^{\mathrm{b}}$ and \\ Reiko Oda* ${ }^{\mathrm{e}}$
}

We demonstrate the direct induction of chirally arranged organic dye-linked polysilsesquioxane through a sol-gel transcription using a chiral supramolecular template. The chiral arrangement was confirmed by electronic and vibrational circular dichroism and circularly polarized luminescence spectroscopies.

For the fabrication of chiral inorganic materials, one of the representative approaches is the sol-gel transcription method using the imprinting of chiral organic templates with achiral inorganic precursors. Such inorganic structures can then be used as a template to organize achiral dyes to induce chiroptical properties. ${ }^{1}$ We have previously established the preparation procedure of chirally twisted and helical nanoribbons of inorganic silica from self-assembled gemini surfactant $^{1 \mathrm{a}, 1 \mathrm{~b}, 2}$ and have found that they show a large vibrational circular dichroism (VCD) signal at around 1000$1150 \mathrm{~cm}^{-1}$, indicating that the siloxane network was chirally arranged. ${ }^{2 \mathrm{~d}}$ This is a rare example in which the chiral arrangement of the inorganic siloxane network was clearly evidenced by VCD spectroscopy. This chirality induction comes from the chiral surface of the organic templates, to originally achiral inorganic ones.

Such chiral silica can potentially be used for enantioselective separation/recognition ${ }^{3}$ and have the ability to induce chiral arrangement to the achiral molecules adsorbed on their

\footnotetext{
Materials Development Department, Kumamoto Industrial Research Institute

3-11-38 Higashimachi, Higashi-ku Kumamoto 862-0901 (Japan)

E-mail: nagaoka@kmt-iri.go.jp

Department of Applied Chemistry and Biochemistry, Kumamoto University

2-39-1 Kurokami, Chuo-ku Kumamoto 860-8555 (Japan)

E-mail: ihara@kumamoto-u.ac.jp

International Research and Education Centre of Advanced Energy Science,

Graduate School of Energy Science, Kyoto University

Yoshida-Honmachi, Sakyo-ku Kyoto 606-8501 (Japan)

d. Institut des Sciences Moléculaires (UMR5255 ISM), CNRS, Université de Bordeaux

351 Cours de la Libération, 33405 Talence (France)

Institut de Chimie \& Biologie des Membranes \& des Nano-objets (UMR5248

$C B M N), C N R S$, Université de Bordeaux, Institut Polytechnique Bordeaux

2 rue Robert Escarpit, 33607 Pessac (France)

E-mail: roda@iecb.u-bordeaux.fr

+ Footnotes relating to the title and/or authors should appear here.

Electronic Supplementary Information (ESI) available: [Experimental details and

Supplementary figures]. See DOI: 10.1039/x0xx00000x
}

surface. ${ }^{4}$ To further enhance its functionality and broaden its applicability, introducing organic functional groups or molecules is an effective strategy. They are generally introduced using monosilylated achiral organic molecules [X$\mathrm{Si}(\mathrm{OR})_{3}: \mathrm{X}=$ organic functional group/molecule, $\mathrm{R}=\mathrm{CH}_{3}$ or $\mathrm{C}_{2} \mathrm{H}_{5}$ ] by post-synthesis grafting on the surface of chiral silica. Such organic functional groups/molecules can show chiral properties. ${ }^{5}$ The organic-functionalized moieties have been, however, limited only on the silica surfaces, and the concentration of the functional groups/molecules is thus low on the whole.

Multisilylated organic molecules $\left[\mathrm{X}-\left(\mathrm{Si}(\mathrm{OR})_{3}\right)_{n}: \mathrm{n} \geq 2\right]$ are interesting alternatives as precursors to fabricate organic functionalized silica (strictly, polysilsesquioxane) with a high concentration of organic groups/molecules. In particular, the organic dye-functionalized silica shows interesting optoelectronic properties ${ }^{6}$ due to the densely ordered and immobilized organic dye molecules inside polysilsesquioxane networks. However, the organic dye-linked polysilsesquioxane structures from achiral precursor alone without any chiral dopants that show chiroptical signals have never been reported.

In this paper, we demonstrate the fabrication of organic dye biphenyl-linked polysilsesquioxane (hereafter, abbreviated as BP-PSQ) through the sol-gel transcription using achiral precursor, bis-triethoxysilylated biphenyl (hereafter, abbreviated as BS-BP, Fig. 1a), with a chiral organic template. For this procedure, a water-miscible organic liquid containing a small amount of water is used for the sol-gel transcription to dissolve water-insoluble BS-BP and to hydrolyse its ethoxysilyl

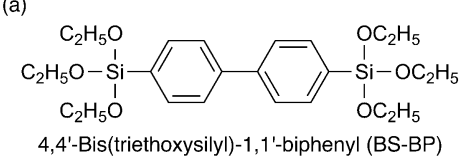

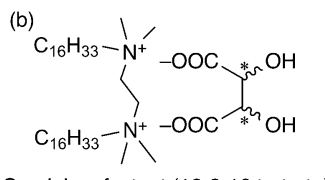

Gemini surfactant (16-2-16 tartrate)
Fig. 1 Chemical structure of (a) biphenyl-linked silsesquioxane precursor and (b) cationic gemini surfactant with enantiomeric tartrate counterion used in this study. 
groups. We have previously shown that the cationic gemini surfactant with an enantiomeric tartrate counterion (hereafter, abbreviated as 16-2-16 tartrate, Fig. 1b) keep their chiral organization both in water and in pyridine as the main solvent, ${ }^{2 a}$ and furthermore, can be used as a template for the sol-gel transcription. ${ }^{1 \mathrm{a}, 1 \mathrm{~b}, 2}$

We first investigated the assembling morphology of 16-2-16 tartrate in pyridine containing water. The pyridine:water ratio was set at 19:1 v/v. 16-2-16 tartrate (20 mM) was dissolved in this mixture by heating to $80{ }^{\circ} \mathrm{C}$. After cooled to $20{ }^{\circ} \mathrm{C}$, the solution immediately became a transparent gel (Fig. S1a) similarly to that in pure pyridine. The gel-sol transition was visually observed upon heating and the transition temperature $\left(T_{\text {gel }}\right)$ was detected using differential scanning calorimetry (DSC) and was estimated to be $52{ }^{\circ} \mathrm{C}$ (transition enthalpy, $\Delta H=$ $1.3 \mathrm{~kJ} \mathrm{~mol}^{-1}$ ) in the heating process (Fig. S1c). When the concentration was decreased to $5.0 \mathrm{mM}$, only a weak gel (Fig. S1b) was observed. Low-voltage scanning transmission electron microscopy (LV-STEM, $30 \mathrm{kV}$ ) observation revealed that 16-2-16 tartrate self-assembled and formed twisted

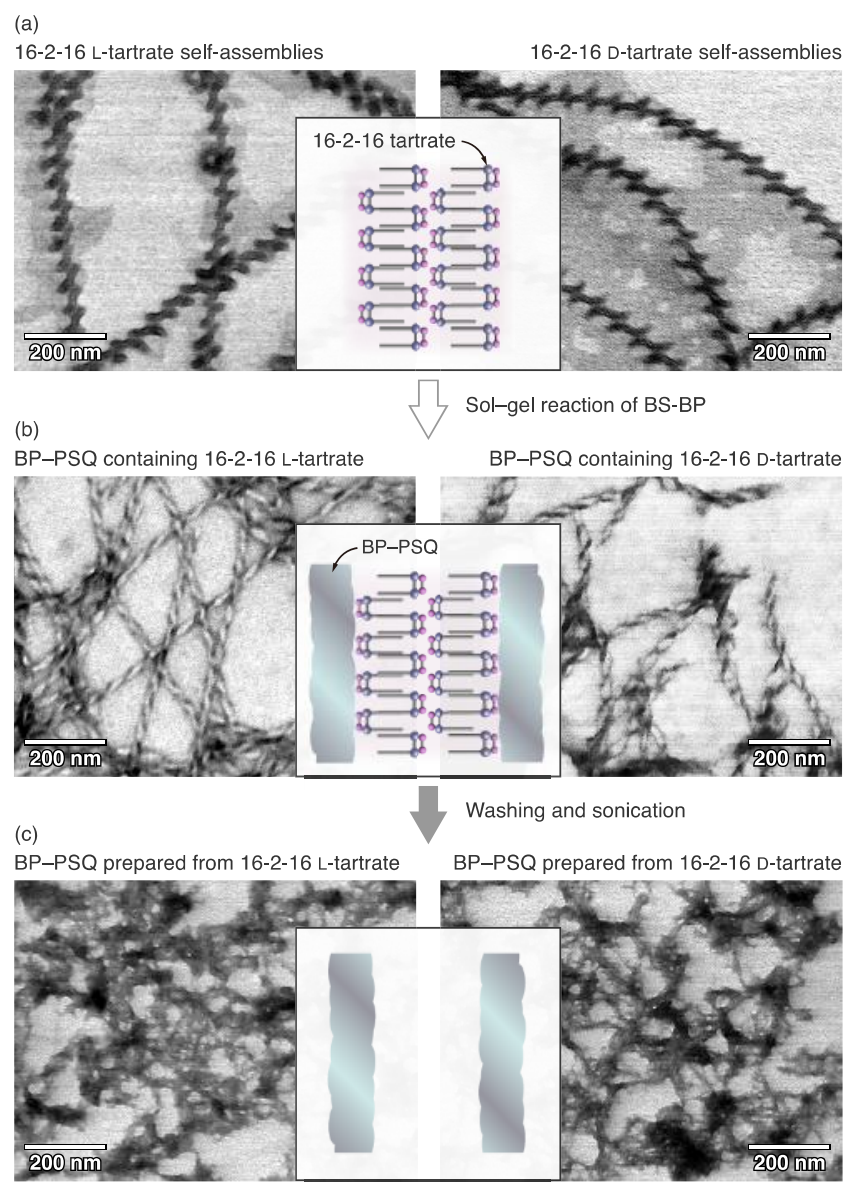

Fig. 2 LV-STEM images of (a) 16-2-16 tartrate self-assemblies in the cast film prepared from a $5.0 \mathrm{mM}$ pyridine-water mixture $(19: 1 \mathrm{v} / \mathrm{v})$ post-stained with $\mathrm{OsO}_{4}$, (b) unstained BP-PSQ containing 16-2-16 tartrate assemblies, and (c) unstained BPPSQ after washing and sonication; left: prepared from 16-2-16 L-tartrate, right: prepared from 16-2-16 D-tartrate. Inset shows schematic illustrations of each cross-sectional surface. ribbon-like nanostructures in the pyridine-water mixture (19:1 v/v). 16-2-16 L-tartrate formed exclusively right-handed twisted ribbons (Fig. 2a, left), and its enantiomer, 16-2-16 Dtartrate, formed left-handed ones (Fig. 2a, right). These nanoribbons had a width of $18 \pm 2 \mathrm{~nm}$ and a pitch of $107 \pm 12$ $\mathrm{nm}$ on the average and were strongly entangled and bundled. The handedness and width are similar to their assemblies in water. ${ }^{7}$

These twisted nanoribbons were then used as the templates in the sol-gel reaction of BS-BP. In a typical procedure, BS-BP (20 $\mu \mathrm{L})$ and $n$-butylamine ( $20 \mu \mathrm{L}$, as a catalyst) were added to the partial gel of 16-2-16 tartrate $(5.0 \mathrm{mM}$, aged for $24 \mathrm{~h}$ at 20 $\left.{ }^{\circ} \mathrm{C}\right)$ in a pyridine-water mixture $(4 \mathrm{~mL}, 19: 1 \mathrm{v} / \mathrm{v})$ and thoroughly mixed with a vortex mixer. After keeping at $20^{\circ} \mathrm{C}$ for $24 \mathrm{~h}$, precipitates were formed which consist of BP-PSQ containing 16-2-16 tartrate. The LV-STEM observation of these precipitates revealed that they maintained twisted ribbon-like nanostructures (width: $23 \pm 4 \mathrm{~nm}$, pitch: $123 \pm 10 \mathrm{~nm}$, Figs. $2 \mathrm{~b}$ and S2). The slight increase in the pitch may be due to the destabilisation of chiral structure upon transcription as observed previously. ${ }^{2 \mathrm{~b}}$ The BP-PSQ containing 16-2-16 tartrate was then washed with hot methanol $\left(55{ }^{\circ} \mathrm{C}\right)$ in order to remove the template molecules along with the excess precursor, catalyst, and solvent. The last washing solution hardly showed any peak in UV-vis absorption spectra (Fig. S3), indicating that the template and other molecules were almost completely removed from BP-PSQ. The washed BP-PSQ was sonicated in ethanol for good dispersion. Fig. 2c shows LVSTEM images of the dispersed BP-PSQ where only poorly structured nanofiber-like structures can be seen and the twisted ribbon-like nanostructures are destroyed after washing and sonication, unlike the silica nanoribbons prepared from tetraethyl orthosilicate from the same gemini surfactant chiral self-assemblies in water ${ }^{1 \mathrm{a}, 2 c, 2 \mathrm{~d}}$ and other organosilica materials prepared from BS-BP by other researchers. ${ }^{6 b, 8}$ This suggests that BP-PSQ walls prepared by this method are more fragile than silica. Indeed, the twist ribbon did not survive even by simply washing under "soft" condition without sonication or simple dilution with pyridine. Fig. 3a shows the powder X-ray diffraction (XRD) pattern of the washed and sonicated BP-PSQ after freeze-drying. Five diffraction peaks are clearly visible. The four peaks at $7.6,15.6,22.8$, and $30.6^{\circ}$ had the ratio of about 1:2:3:4, indicating periodic structure with $\sim 1.17 \mathrm{~nm}$
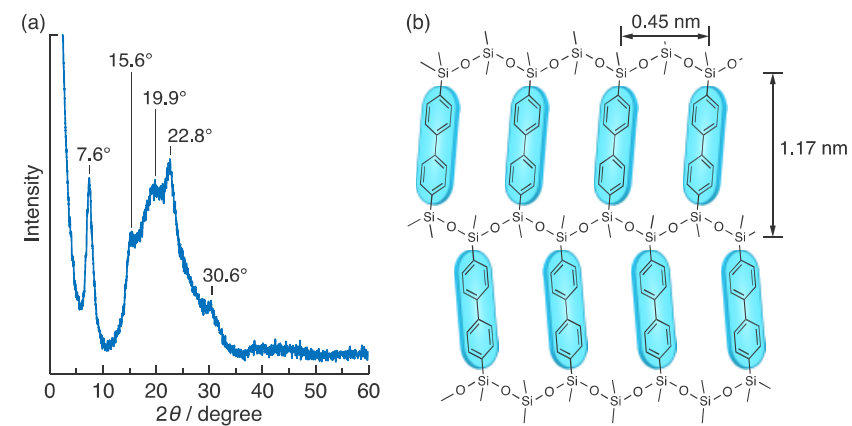

Fig. 3 (a) Powder XRD pattern and (b) schematic illustration of BP-PSQ prepared from 16-2-16 L-tartrate. 
periodicity. ${ }^{8 a}$ This may show that BP-PSQ forms a molecularscale lamellar structure as illustrated in Fig. $3 \mathrm{~b}$. The diffraction peak at $19.9^{\circ}(\sim 0.45 \mathrm{~nm})$ may exhibit the distance between the neighbouring biphenyl moieties which are stacked with each other. These values are consistent with the result of the simulated structure (Fig. S4). The broad peak centred at around $20^{\circ}$ is probably due to the amorphous silsesquioxane.

These results indicate the much lower cross link density of BP-PSQ than that of silica, which may lead to more fragile network, which may explain why the mesoscopic helical structure does not resist washing and sonication. The washed and sonicated BP-PSQ dispersed in ethanol $(0.05 \mathrm{mM}$ as biphenyl-bis(silsesquioxane) unit) was subjected to ultravioletvisible (UV-vis) absorption, fluorescence, electronic circular dichroism (ECD), and circularly polarized luminescence (CPL) measurements, and the obtained spectra are shown in Fig. 4. Compared to its precursor, BS-BP $(0.05 \mathrm{mM})$ molecularly dispersed solution in ethanol which has an absorption peak at $261 \mathrm{~nm}$ (Fig. 4a), the absorption peak of the BP-PSQ decreased and slightly red-shifted to $263 \mathrm{~nm}$ (Fig. 4a). These results imply that the biphenyl moieties of the BP-PSQ interact each other in the ground state which is in agreement with the $X R D$ result. When excited at absorption maximum wavelength, compared to the emission band of BS-BP (emission maximum wavelength, $\lambda_{\mathrm{em}}=312 \mathrm{~nm}$; full width at half maximum, FWHM $=3360 \mathrm{~cm}^{-1}(34 \mathrm{~nm})$; Stokes shift $\left.=6260 \mathrm{~cm}^{-1}(51 \mathrm{~nm})\right)$, the
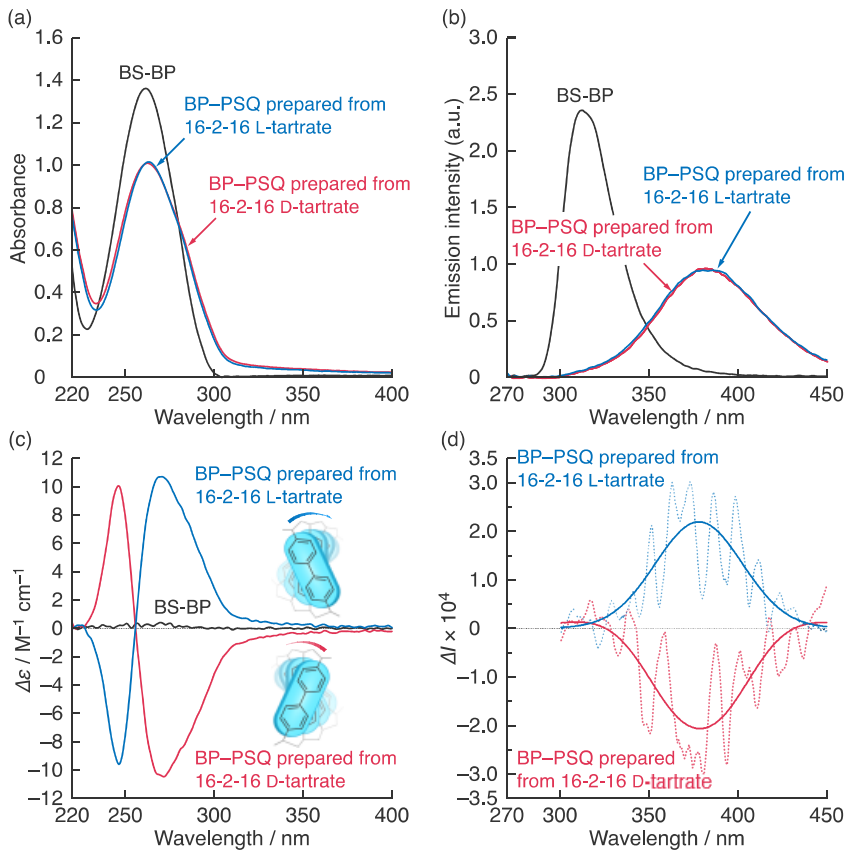

Fig. 4 (a) UV-vis absorption, (b) fluorescence, (c) ECD and (d) CPL spectra of BS-BP $(0.05 \mathrm{mM})$ and BP-PSQ $(0.05 \mathrm{mM}$ as biphenyl-bis(silsesquioxane) unit) prepared from 16-2-16 Land D-tartrate in ethanol at $20^{\circ} \mathrm{C}$; path length $=1.0 \mathrm{~cm}$ (except for (b)). For (c) and (d), the BS-BP and BP-PSQ were excited at 261 and $263 \mathrm{~nm}$, respectively. The CPL spectra in (d) were fitted using Voigt functions and the original spectra are shown as dotted lines. Inset in (c) shows schematic illustration of chirally arranged biphenyl moieties of BP-PSQ.
BP-PSQ showed a broader emission band at longer wavelength $\left(\lambda_{\mathrm{em}}=384 \mathrm{~nm}, \mathrm{FWHM}=4840 \mathrm{~cm}^{-1}(71 \mathrm{~nm})\right.$, Stokes shift $\left.=12000 \mathrm{~cm}^{-1}(121 \mathrm{~nm})\right)$ without any peaks and shoulders around $312 \mathrm{~nm}$ (Fig. 4b). In addition, the emission of the BPPSQ has a longer lifetime $(\tau)$ than that of BS-BP $\left(\tau_{\mathrm{BP}-\mathrm{PSQ}}=27 \mathrm{~ns}\right.$, $\tau_{\mathrm{BS}-\mathrm{BP}}=5 \mathrm{~ns}$, Fig. S5). A significant red shift and broadening of an emission band and a lengthened fluorescence lifetime are typical behaviour that appears as polycyclic aromatic dyes (pyrene, anthracene, and so on) form excimers (excited dimers), whereas excimer formation is generally difficult for typical biphenyl species because of the steric hindrance of the twisted phenyl rings. Thus, it seems that the excimer formation of the biphenyl moieties of the BP-PSQ is enabled due to the dense packing structure through the polysilsesquioxane network. On the other hand, in general, excimer formation causes a decrease in the fluorescence quantum yield $(\phi)$ because of the strong interaction between dye molecules in the excited state. In the present case, it is worth noting that in spite of the excimer formation, the BPPSQ has a higher quantum yield than that of its precursor $\left(\phi_{\mathrm{BP}-}\right.$ PSQ $=0.31, \phi_{\mathrm{BS}-\mathrm{BP}}=0.23$ ). This enhancement of the quantum yield can be explained by the restricted internal rotation of the densely packed biphenyl moieties, caused by the intermolecular steric hindrance. Interestingly, the BP-PSO showed a Cotton effect (ECD signal) in the region of the absorption band and CPL in the region of the emission band with a mirror image between the BP-PSQ prepared from 16-216 L- and D-tartrate (Figs. 4c and d) even though it has no asymmetric atom in the molecule and chiral shape. The dissymmetry factors ( $g$-factors) were estimated to be $17.8 \times$ $10^{-4} \mid$ and $\left|5.6 \times 10^{-4}\right|$ for the ECD signals at 246 and $271 \mathrm{~nm}$, respectively, and $\left|2.1 \times 10^{-4}\right|$ for the CPL at $378 \mathrm{~nm}$. These results indicate that in both ground and excited states, the biphenyl moieties of the BP-PSQ were chirally arranged as illustrated in the inset of Fig. 4c, that is, right-handed arrangement for the BP-PSQ prepared from 16-2-16 L-tartrate and left-handed arrangement for the one prepared from 16-216 D-tartrate according to exciton chirality theory, ${ }^{9}$ which means that the gemini surfactant-based chirality was successfully transferred to the BP-PSQ at the bonding network level. While longer aging time $(3 \mathrm{~d})$ during sol-gel process caused an increase in the yield of the BP-PSQ, the ECD intensity was decreased (Fig. S6). This suggests that as the BPPSQ wall grows, the internal chiral order is gradually lost.

In general, chirally arranged dye molecules in media tend to be disrupted by heating because in many cases, the chiral structures are formed and maintained by weak intermolecular forces such as Van der Waals force, hydrogen bonds, and hydrophobic effect. ${ }^{10}$ In the present system, the chiral arrangement showed very little modification upon heating up to $70^{\circ} \mathrm{C}$ (Fig. S7) as demonstrated by the ECD signal of the biphenyl moieties of the BP-PSQ. Moreover, the chiral arrangement remained almost unchanged even after standing at $20{ }^{\circ} \mathrm{C}$ in the dark for a month (Fig. S8). The chiral arrangement of biphenyl moieties is clearly stabilized into the inorganic polysilsesquioxane network although the mesoscopic chiral shape is lost. 

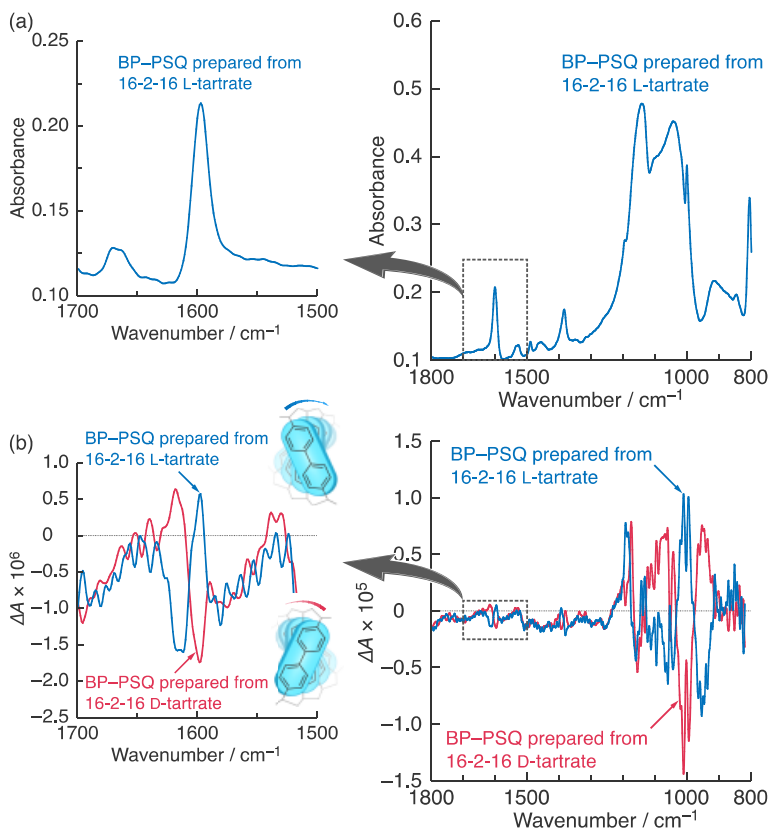

Fig. 5 (a) IR and (b) VCD spectra of freeze-dried BP-PSQ prepared from 16-2-16 L- and D-tartrate. Inset in (b, left) shows schematic illustration of chirally arranged biphenyl moieties of BP-PSQ.

The chirality of the polysilsesquioxane network moiety was also investigated. Fig. 5 shows infrared (IR) and VCD spectra of freeze-dried BP-PSQ. The IR spectrum showed a complex large absorption band in the $1250-950 \mathrm{~cm}^{-1}$ spectral range, with peaks at 1045 and $1143 \mathrm{~cm}^{-1}$ (Fig. 5a, right), which are associated with the $\mathrm{Si}-\mathrm{O}-\mathrm{Si}$ asymmetric stretching vibration $\left(v_{\text {as }} \mathrm{Si}-\mathrm{O}-\mathrm{Si}\right){ }^{11}$ This multicomponent broad band reveals VCD signatures which are mirror image for the two enantiomeric structures (Fig. 5b, right). These results suggest that the polysilsesquioxane network is also chirally arranged. Furthermore, a small, but clear bisignate Cotton effect (VCD signal) appeared around $1600 \mathrm{~cm}^{-1}$, related to the $v C=C$ stretching vibration of the phenyl rings, with a mirror image between the BP-PSQ prepared from 16-2-16 L- and D-tartrate (Fig. 5b, left), indicating the chiral arrangement of the biphenyl moieties. Importantly, the sign of the VCD signal, namely the direction of the chiral arrangement of the biphenyl moieties, ${ }^{12}$ is consistent with the result of the ECD spectra.

In conclusion, we succeeded in inducing the chiroptical signals from organic dye-linked polysilsesquioxane through chirality transfer from supramolecular templates during solgel transcription process. Although washing and sonication destroy the macroscopic twisted morphology, local chiral organization of organic dye moieties inside the polymerized network was well preserved. This is the first example of the organic-linked polysilsesquioxane fabricated from achiral precursor alone (without any chiral dopants) that shows obvious chiral optical signals (ECD, CPL, and VCD). These findings provide a new promising strategy for the design of organic-inorganic chiral hybrid materials. The details on the mechanism on the chirality induction from chiral self- assemblies to BP-PSQ polymerisation is under investigation.

All authors discussed the results, commented on the manuscript and contributed to the interpretation of the data. The gemini surfactant was synthesized by N.R. and H.Y. The preparation condition of BP-PSQ was established by N.R. and T.K. DSC was measured by N.R. STEM and SEM observations were carried out by N.R. and H.Y. XRD was measured by H.Y. and S.N. Molecular simulation was carried out by T.S. UV-vis absorption, fluorescence and ECD spectra were measured by N.R. and T.K. CPL spectra was measured by Y.O. $\tau$ was measured by K.Y. $\phi$ was measured by H.Y. VCD spectra was measured by T.B. and K.Y. N.R., H.I. and R.O. designed the study. S.N. and M.T. were involved in study design. N.R. drafted the manuscript and Y.O., H.I. and R.O. assisted in the preparation of the manuscript.

This work was supported by "Nippon Sheet Glass Foundation for Materials Science and Engineering" and "French-Japanese International Associated Laboratory, Chiral Nanostructures for Photonic Applications (LIA-CNPA)".

\section{Conflicts of interest}

There are no conflicts to declare.

\section{Notes and references}

1 (a) N. Ryu, Y. Okazaki, K. Hirai, M. Takafuji, S. Nagaoka, E. Pouget, H. Ihara and R. Oda, Chem. Commun., 2016, 52, 5800; (b) Y. Okazaki, N. Ryu, T. Buffeteau, S. Nagaoka, E. Pouget, S. Nlate, H. Ihara and R. Oda, Chem. Commun., 2018, 54, 10244; (c) S. Tsunega, R.-H. Jin, T. Nakashima and T. Kawai, ChemPlusChem, 2019, 84, 1.

2 (a) K. Sugiyasu, S.-i. Tamaru, M. Takeuchi, D. Berthier, I. Huc, R. Oda and S. Shinkai, Chem. Commun., 2002, 1212; (b) T. Delclos, C. Aimé, E. Pouget, A. Brizard, I. Huc, M.-H. Delville and R. Oda, Nano Lett., 2008, 8, 1929; (c) Y. Okazaki, J. Cheng, D. Dedovets, G. Kemper, M.-H. Delville, M.-C. Durrieu, H. Ihara, M. Takafuji, E. Pouget and R. Oda, ACS Nano, 2014, 8, 6863; (d) Y. Okazaki, T. Buffeteau, E. Siurdyban, D. Talaga, N. Ryu, R. Yagi, E. Pouget, M. Takafuji, H. Ihara and R. Oda, Nano Lett., 2016, 16, 6411.

3 (a) P. Paik, A. Gedanken and Y. Mastai, ACS Appl. Mater. Interfaces, 2009, 1, 1834; (b) H. Qiu, Y. Inoue and S. Che, Angew. Chem. Int. Ed., 2009, 48, 3069; (c) P. Romero, S. Lacasta, V. Sebasti, C. Casado, E. Vispe, L. Pilar, S. Uriel and J. Coronas, Chem. Mater., 2011, 23, 1280.

4 H. Matsukizono and R.-H. Jin, Angew. Chem. Int. Ed., 2012, 51, 5862.

5 T. Yokoi, S. Sato, Y. Ara, D. Lu, Y. Kubota and T. Tatsumi, Adsorption, 2010, 16, 577.

6 (a) H. Takeda, Y. Goto, Y. Maegawa, T. Ohsuna, T. Tani, K. Matsumoto, T. Shimada and S. Inagaki, Chem. Commun., 2009, 6032; (b) S. Inagaki, O. Ohtani, Y. Goto, K. Okamoto, M. Ikai, K.-i. Yamanaka, T. Tani and T. Okada, Angew. Chem. Int. Ed., 2009, 48, 4042; (c) N. Mizoshita, M. Ikai, T. Tani and S. Inagaki, J. Am. Chem. Soc., 2009, 131, 14225.

7 R. Oda, I. Huc, M. Schmutz, S. J. Candau and F. C. MacKintosh, Nature, 1999, 399, 566.

8 (a) M. P. Kapoor, Q. Yang and S. Inagaki, J. Am. Chem. Soc., 2002, 124, 15176; (b) S. Fujita and S. Inagaki, Chem. Mater., 2008, 20, 891; (c) Y. Goto, N. Mizoshita, O. Ohtani, T. Okada, 
T. Shimada, T. Tani and S. Inagaki, Chem. Mater., 2008, 20, 4495.

9 (a) M. Chang, H. V. Meyers, K. Nakanishi, M. Ojika, J. H. Park, M. H. Park, R. Takeda, J. Vázquez and W. T. Wiesler, Pure. Appl. Chem., 1989, 61, 1193; (b) N. Berova and K. Nakanishi, in Circular Dichroism: Principles and Applications, ed. N. Berova, K. Nakanishi and R. Woody, Wiley-VCH, New York, 2nd edn, 2000; ch. 12, pp. 337-382; (c) L.-C. Lo, C.-T. Yang and C.-S. Tsai, J. Org. Chem., 2002, 67, 1368.

10 (a) M. Takafuji, A. Ishiodori, T. Yamada, T. Sakurai and H. Ihara, Chem. Commun., 2004, 1122; (b) N. Ryu and H. Hachisako, Chem. Lett., 2015, 44, 211; (c) N. Ryu, Y. Okazaki, E. Pouget, M. Takafuji, S. Nagaoka, H. Ihara and R. Oda, Chem. Commun., 2017, 53, 8870.

11 M. Handke, B. Handke, A. Kowalewska and W. Jastrzȩbski, J. Mol. Struct., 2009, 924-926, 254.

12 T. Taniguchi and K. Monde, J. Am. Chem. Soc., 2012, 134, 3695. 